there was evidence of NLRP3 inflammasome priming for both endothelial cell types, only EA.hy926 cells showed extracellular IL-1 $\beta$ production. Other end products of NLRP3 inflammasome activation may be worthy of investigation in order to fully describe the response of endothelial cells to innate stimuli.

\section{P39 POST-MYOCARDIAL INFARCTION EXTRACELLULAR MATRIX REMODELLING IN THE PIG IS ASSOCIATED WITH ALTERED EXPRESSION OF MICRO-RNAS}

A Harvard, H Dobrzynski, AJ Atkinson, A D'Souza, J Yanni, M Petkova, B Borbas, N Malik, CM Holt. Division of Cardiovascular Sciences, University of Manchester

\subsection{6/heartjnl-2018-BSCR.44}

Extracellular matrix remodelling is a key component of myocardial repair after ischaemic injury, and contributes to fibrotic changes leading to heart failure. MicroRNAs, short non-coding RNAs which regulate gene expression, have been shown to be dysregulated in a number of rodent models of ischaemia and heart failure, yet limited data is available in large translational animal models.
The expression of a number of fibrosis-associated microRNAs were investigated in a relevant porcine model of mild to moderate left ventricular (LV) dysfunction induced by coronary microembolisation ( $n=3$ MI and $n=3$ control pigs). Samples of myocardial tissue were obtained 4 weeks post-MI and classified into infarct, border and remote (normal) regions. The infarct area was histologically mostly acellular with dense inflammatory infiltrate, extracellular matrix expansion and collagen deposition as seen with Masson's trichrome and Picrosirius red staining. qRT-PCR analysis of microRNA expression demonstrated downregulation of miR-133a $(p=0.0085)$, whereas miR-214 was upregulated $(p=0.0027)$ in the infarct region, compared to normal/control myocardium.

miR-133a has previously been shown to protect against cardiac fibrosis, and is downregulated in the heart in numerous small-animal models of heart failure. miR-214 is upregulated in the fibrotic heart, and its inhibition has been shown to be protective against hepatic fibrosis. Therefore, our porcine data supports the hypothesis that pathological myocardial remodelling may be influenced by altered expression of microRNAs, and confirms the pattern of microRNA expression observed in other species. Future work is aimed at testing therapeutic approaches targeting these specific microRNAs. 